\title{
Linx
}

Revue des linguistes de l'université Paris X Nanterre

$45 \mid 2001$

Invariants et variables dans les langues. Études typologiques

\section{Passif et moyen : la structure argumentale des prédicats dans les langues à base intransitive dominante (l'exemple du Hindi/Ourdou)}

\section{Annie Montaut}

\section{OpenEdition}

\section{Journals}

Édition électronique

URL : http://journals.openedition.org/linx/750

DOI : $10.4000 /$ linx.750

ISSN : 2118-9692

\section{Éditeur}

Presses universitaires de Paris Nanterre

Édition imprimée

Date de publication : 1 décembre 2001

Pagination : 83-93

ISSN : 0246-8743

\section{Référence électronique}

Annie Montaut, «Passif et moyen : la structure argumentale des prédicats dans les langues à base intransitive dominante (l'exemple du Hindi/Ourdou)», Linx [En ligne], 45 | 2001, mis en ligne le 20 juin 2012, consulté le 19 avril 2019. URL : http://journals.openedition.org/linx/750 ; DOI : 10.4000/linx.750

Ce document a été généré automatiquement le 19 avril 2019

Département de Sciences du langage, Université Paris Ouest 


\title{
Passif et moyen : la structure argumentale des prédicats dans les langues à base intransitive dominante (l'exemple du Hindi/ Ourdou)
}

\author{
Annie Montaut
}

1 La description traditionnelle du passif est peu probante pour les données hindi / ourdou, tant sur le plan syntaxique (non promotion de l'objet, passivation des intransitifs), que sémantique et pragmatique (l'occultation de l'agent n'est que formelle). C'est avec la classe des intransitifs médio-passifs que le passif semble en opposition tranchée (section 1). L'observation du comportement contrasté de ces deux formes, notamment dans les constructions où elles sont porteuses de valeurs modales (sections 2,3), permet de dégager la spécificité du passif en hindi, mais aussi le caractère déterminant des traits sémantiques associés aux arguments des bases verbales morphologiquement corrélées, dans une langue où les bases transitives sont généralement dérivées des intransitifs ${ }^{1}$.

\section{La construction passive en hindi/ourdou}

2 Le passif hindi moderne est une forme composée (participe accompli, identique à celle du passé simple + auxiliaire « aller », jânâ, dont le passé simple est irrégulier gayâ) ${ }^{2}$.

\subsection{Périphérisation de l'agent sans promotion de l'objet}

Lorsque l'agent est exprimé, la diathèse passive (1b) semble inverser la diathèse active transitive (1a), comme la diathèse ergative (1c), faisant du patient le pivot morphosyntaxique de l'énoncé, qu'il soit défini (1) ou indéfini (2): 


\begin{tabular}{|c|c|}
\hline \multirow[t]{2}{*}{ (1)a } & pulis cor pakRâegî \\
\hline & police-fs voleur-ms attraper-fut-fs \\
\hline & la police attrapera le voleur \\
\hline \multirow[t]{3}{*}{$(1) \mathrm{b}$} & cor pulis se /kedvârâ pakRâ gayâ \\
\hline & voleur-ms police-fs instr /agentPass attraper Pass-aor-ms \\
\hline & le voleur a été attrapé par la police \\
\hline \multirow[t]{3}{*}{$(1) c$} & pulis ne cor pakRâ \\
\hline & police-fs erg voleur-ms attraper-aor-ms \\
\hline & la police a attrapé le voleur \\
\hline \multirow[t]{3}{*}{ (2) } & ek mahilâ âtânkvâdiyon dvârâ mârî gầ \\
\hline & Un femme-fs terroristes par tuer Pass-aor-fs \\
\hline & une femme a été tuée par les terroristes \\
\hline
\end{tabular}

4 Un patient marqué (+ko : postposition dat/acc) s'il est humain ou spécifique entraîne aussi un défaut d'accord parallèle à l'ergatif (3a) et au passif (3b) :

\begin{tabular}{|l|l|l|l|l|l|l|}
\hline (3)a & pulis & ne & tînon & ko & \multicolumn{1}{l|}{ mârâ } \\
\hline \hline & police-fs & erg & trois-p & acc & tuer-aor-ms \\
\hline & La police les a tués tous les trois & \\
\hline (3)b & (pulis & se) & tînon & ko & mârâ & gayâa \\
\hline \hline & (police & instr) & trois & acc & tuer & Pass-aor-ms \\
\hline & tous les trois ont été tués (par la police) \\
\hline
\end{tabular}

5 Si la position de l'agent dans l'ordre non marqué est variable au passif $(1,3)$, elle est toujours initiale dans l'énoncé ergatif non marqué comme dans l'énoncé transitif inaccompli (1a). Le passif est possible à tous les temps et aspects alors que l'ergatif ne l'est qu'à l'accompli. Enfin, l'agent du passif est rarement exprimé alors que celui de l'ergatif n'est omis que si le contexte permet de le récupérer. A la possibilité d'indétermination de l'agent, s'ajoute celle, au passif, par le jeu des postpositions, de représenter un agent indirectement ( $k e d v a ̂$ âa $)$ ou directement impliqué (se). Les deux structures ne sont donc pas redondantes, la construction passive seule constituant un véritable dispositif d'occultation de l'agent. Mais elle n'entraîne pas systématiquement la promotion du 
patient: ce dernier peut conserver sa marque d'accusatif (3), mais aussi n'être pas représenté (4).

\begin{tabular}{|l|l|l|l|l|}
\hline (4) & dhamkâyâa gayâ, & mârâ-pîtâa & gayâa \\
\hline \hline & menacer Pass-aor-ms, & frapper-battre & Pass-aor-ms \\
\hline \hline & on la menaça, on la battit (mais elle refusa d'y aller) \\
\hline
\end{tabular}

6 Surtout, un grand nombre de verbes intransitifs sont passivables, soit à des fins d'indétermination du participant unique (5), soit pour exprimer des valeurs dites modales, prescription au présent générique sans agent (6) ou «incapacité » en contexte négatif avec un agent instrumental (7) :

\begin{tabular}{|c|c|c|c|c|c|c|}
\hline (5) & thoRâ & Tahlâ & & \multicolumn{3}{|l|}{ jâe? } \\
\hline & un peu & se-promener & & \multicolumn{3}{|c|}{ Pass-subj-ms } \\
\hline & \multicolumn{6}{|c|}{ si on se promenait un peu? / Faisons quelques pas } \\
\hline \multirow[t]{3}{*}{ (6) } & is & tarah & hansâ & /cillâyâ & nahîn & jâtâ \\
\hline & cette & façon & rire & /crier & neg & Pass-pres-ms \\
\hline & \multicolumn{6}{|c|}{ on ne doit pas rire /crier ainsi } \\
\hline (7) & mujhse & soyâ & nahîn & \multicolumn{3}{|l|}{ gayâ } \\
\hline & je-instr & dormir & neg & \multicolumn{3}{|c|}{ Pass-aor } \\
\hline & \multicolumn{6}{|c|}{ il ne put fermer l'œil (lit. par lui il ne fut pas dormir) ${ }^{3}$} \\
\hline
\end{tabular}

7 La mise à l'arrière plan de l'agent (Shibatani 1985), son occultation Brahim (1996) ne signifient pourtant pas l'absence de pertinence du rôle dans la structure.

\subsection{Propriétés de contrôle de l'agent}

8 C'est l'agent en effet qui contrôle réflexivation et coordination, quand cette dernière s'exprime sous la forme d'un participe conjonctif, lequel requiert un sujet coréférent au « sujet » du verbe principal ${ }^{4}$ :

\begin{tabular}{|l|l|l|l|l|l|}
\hline (8)a & cîzen & usmen & pîskar & Dâlî & gaîn \\
\hline \hline & chose-fp & ceci-dans & broyant & jeter & Pass-aor-fp \\
\hline & \multicolumn{2}{|l}{ on broya les ingrédients et on les mit dedans } \\
\hline
\end{tabular}




\begin{tabular}{|l|l|l|l|l|l|l|l|l|}
\hline$(8) b$ & kyâ & use & apnâ & dRSTikoN & samjhâyâa & nahîn & jâ & saktâ hai $?$ \\
\hline & interr & il-dat & refl & point-de-vue & expliquer & neg & Pass & pouvoir pres-3ms \\
\hline
\end{tabular}

9 L'agent conserve donc nombre de propriétés sémantico-référentielles, comme le montre aussi le fonctionnement des "explicateurs verbaux»: ces verbes semi-auxiliarisés, qui perfectivent le procès en orientant la diathèse (« prendre », le, vers le sujet, « donner », de , vers l'objet) sont sélectionnés comme ils le seraient dans la phrase active, c'est-à-dire en fonction de l'agent (les chasseurs) et non du patient (les singes):

\begin{tabular}{|c|c|}
\hline (9)a & Bandar pakaR lie gae aur gânv se haTâ die gae \\
\hline & $\begin{array}{l}\text { singes attraper prendre Pass-aor et village abl écarter donner } \\
\text { Université Marc Bloch, Strasbourg Pass-aor }\end{array}$ \\
\hline & les singes furent capturés et éloignés du village (on s'empara d'eux et on les expulsa) \\
\hline$(9) \mathrm{b}$ & shikârion ne bandar pakaR lie aur gânv se haTâ die \\
\hline & $\begin{array}{l}\text { chasseurs erg singes attraper prendre-aor et village abl écarter } \\
\text { donner-aor }\end{array}$ \\
\hline & seurs attrapèrent les singes et les éloignèrent du village \\
\hline
\end{tabular}

10 Si ces faits ne permettent pas de conclure à la subjectalité de l'agent, étant plus sémantiques et pragmatiques que strictement syntaxiqueset grammaticaux, ils montrent que le terme le plus élevé dans la hiérarchie des arguments reste l'agent, bien qu'il soit omis la plupart du temps. On ne peut donc expliquer par la périphérisation de l'agent les affinités que les structures passives entretiennent avec l'expression des procès spontanés, comme le suggère Shibatani (1985), dans le prolongement de la valeur potentielle (type 7). C'est en hindi / ourdou, les intransitifs -dont sont dérivés les transitifs ${ }^{5}-$ qui prennent en charge les procès spontanés, et jamais les passifs.

\subsection{Passifs et intransitifs de sens médio-passif}

11 La série (10) illustre la différence entre transitif et intransitif morphologiquement corrélés (ici apophonie et rhotacisme) : la base intransitive Tût signifie «se casser, être cassé », son correspondant transitif toR (à deux places : agent, patient) signifie «casser, briser »

\begin{tabular}{|l|l|l|l|l|}
\hline (10)a & maine & gilâs & \multicolumn{2}{l|}{ toRâa } \\
\hline & je-erg & verre-ms & casser-aor-3ms & 'j'ai cassé le verre' \\
\hline$(10) b$ & gilâs & & TûTâa \\
\hline
\end{tabular}




\begin{tabular}{|l|l|l|l|l|}
\hline & verre-ms & & casser-aor-ms & 'le verre s'est cassé \\
\hline$(10) c$ & gilâs & toRâ & gayâ & 'le verre a été cassé' \\
\hline & verre & casser-tr & Pass-aor & \\
\hline
\end{tabular}

(10b) seul exprime un résultat disjoint de son auteur, par exemple le bris du verre par suite du transport. Comme le se-moyen-passif du français, il ne met pas en scène la responsabilité d'un agent, alors que (10a) l'explicite et (10c) la suppose. L'agent à la source du procès, de la transmission d'énergie pour reprendre la métaphore de Langacker, est dans les trois cas soit périphérisé soit absent, aboutissant à une " image " qui profile le résultat et non, comme le ferait l'énoncé transitif (10d), la source du procès comme « figure principale».

\begin{tabular}{|l|l|l|l|l|}
\hline (10)d & main & gilâs & toRûngâ & \\
\hline \hline & je-nom & verre & casser-fut-1s & 'je casserai le verre' \\
\hline
\end{tabular}

Dans (10a-c), c'est la dernière étape du procès, son résultat en l'occurrence, qui est représenté, disjoint de la source ${ }^{6}$. En quoi passif et ergatif représentent des constructions potentiellement absolues, indépendantes de l'entité cause, plus proches donc des intransitifs que des transitifs. Cependant cette analogie de profilage et de scénario conceptuel entre intransitifs et passif est trompeuse pour le hindi, comme le montrent les oppositions claires entre khulnâ « s'ouvrir/être ouvert » et le passif de kholnâa "ouvrir ", baRhnâ « augmenter, croître » et le passif de baRhânâ « faire croître ».

\begin{tabular}{|l|l|}
\hline$(11) a$ & $b a ̂ l$ khulethe, jo khole nahîn gaethe, \\
\hline & cheveux s'ouvrirppft, rel ouvrir neg Passppft \\
\hline & sirf Dhîlehokar juRe sebâhar nikalâethe \\
\hline & Seulementlacheétant chignon hors.de sortirvenir \\
\hline & $\begin{array}{l}\text { elle avait les cheveux défaits, sans qu'ils aient été défaits, ils étaient seulement sortis de } \\
\text { son chignon }\end{array}$ \\
\hline$(11) b$ & dâRhî baRhîthî, vaise nahîn jise baRhâyâa jâtâhai, \\
\hline & barbe pousserppft, comme neg rel-acc faire-pousser Passpres \\
\hline & balki jaise vah shaivkarnâ bhûlgaeho \\
\hline & $\begin{array}{l}\text { mais comme il raserfaire oublierallersubj } \\
\text { de se raser avait poussé, pas comme celle qu'on fait pousser, mais comme s'il avait oublié }\end{array}$ \\
\hline
\end{tabular}


Les deux structures s'opposent par le trait agent (+volonté, +contrôle conscient), associé à l'argument principal exprimé ou non (passif) ou exclu de la configuration actancielle (intransitif). Les transitifs, et leur passif, comportent un agent en hindi, alors que les intransitifs de sens medio-passif ont pour participant un patient ${ }^{7}$. La même opposition s'observe pour les prédicats verbaux nominaux, entre le passif des transitifs (verbe support transitif faire kar) et l'intransitif (verbe support intransitif « être» ho) :

\begin{tabular}{|l|l|l|l|l|l|l|l|l|l|}
\hline (11)c & prem & kiyâa & nahîn & jâtâ, & vah & to & ho & gayâ & hai \\
\hline \hline & amour & faire & neg & Pass-pres & il & mais & être & aller & pft \\
\hline \multicolumn{6}{|l}{ l'amour ne se décide pas, il advient } \\
\hline
\end{tabular}

\section{Passifs et intransitifs à valeur modale : l'impossibilité (incapacitative meaning)}

Cependant, s'il est vrai que les deux voix s'opposent fortement, il est une construction où les voix active (du verbe intransitif de sens médio-passif) et passive (du verbe intransitif) semblent se confondre : il s'agit de la construction (7) dite d'incapacité, où l'agent est toujours exprimé à l'instrumental se (et non *ke dvârâ), en contexte toujours négatif ou paranégatif (interrogation, hypothétique, virtuel) ${ }^{8}$.

\subsection{Les faits : parallélismes syntaxiques et sémantiques}

16 La construction dite incapacitative de type (7) concerne aussi les verbes transitifs (12b), entrant ainsi en parallèle avec la construction intransitive de même valeur modale (12a) ${ }^{9}$ :

\begin{tabular}{|l|l|l|}
\hline (12)a & bâhar sedarvâzâa khînclo, sânkal mujhse nahîn khul rahî hai \\
\hline & dehors parporte tireprends, chaîne je-instr neg s'ouvrir progr pres \\
\hline & tire la porte de l'extérieur, je n'arrive pas à (ne suis pas foutu) ouvrir la chaîne \\
\hline (12)b & mujhse darvâzâa kholâ nahîn gayâa \\
\hline & je-instr porte ouvrir neg Pass-aor \\
\hline & je ne pus (me résoudre à) ouvrir la porte \\
\hline
\end{tabular}

17 Cette apparente commutabilité, y compris dans le strict contrôle de la coréférence ${ }^{10}$, infirme-t-elle le contraste développé en 1.3 ? L'agent instrumental en contexte négatif produit-il la même valeur avec un passif (d'intransitif ou de transitif, de verbe simples ou de locutions verbales) qu'avec un intransitif de sens médio-passif? 


\subsection{Divergence entre passif et intransitif médio-passif : structure argumentale et rôles sémantiques}

\subsubsection{Deux types d'intransitifs}

On a souvent remarqué que tous les intransitifs ne sont pas passivables : hansnâa « rire ", muskurânâ « sourire », sonâ « dormir/s'endormir », cillânâ « crier », nâcnâ « danser », jânâa «aller », calnâ « marcher », dauRnâ « courir », etc. le sont, alors que pahuncnâ « arriver », girnâ « tomber ", biknâ « se vendre ", TûTnâa « se casser », kaTnâ « se couper ", bannâ « se fabriquer/être fabriqué ", paknâ « cuire/être cuit » ne le sont pas. Les premiers peuvent se mettre à l'impératif mais non les seconds ${ }^{11}$. Que les premiers correspondent aux verbes d'activité (Vendler, Van Valin), ou aux inaccusatifs, ou que la distinction pertinente soit ailleurs que dans la sémantique verbale, il semble que les intransitifs aptes à entrer dans la construction modale partagent avec les transitifs le fait de comporter un agent dans leur structure argumentale. Cela est d'autant plus clair que, si on parvient à ajouter le trait d'agentivité à des verbes réputés non passivables comme girnâ «tomber», ils admettent le passif. Par exemple, imaginons un entraînement de parachutistes où on jeu où le but de l'opération soit de tomber, un gamin horrifié qui par exemple refuse de jouer le jeu parce qu'il a trop peur ou qu'il trouve ça révoltant, trop nul, trop sale par terre, etc. et on peut avoir un énoncé du genre de (13) :

\begin{tabular}{|l|l|l|l|l|l|l|}
\hline (13) & nahîn, & nahîn, & mujhse & girâ & nahîn & jâegâa \\
\hline & non & non & je-instr & tomber & neg & Pass-fut-3ms \\
\hline & \multicolumn{6}{|l}{ non, c'est impossible, je n'arriverai pas à tomber, je ne peux pas faire ça } \\
\hline
\end{tabular}

Mohanan (1994: 158) fait la remarque pertinente que les verbes comme Darnâ «avoir peur ", ghabrânâ «s'inquiéter ", n'admettant ni l'impératif ni le passif à l'affirmatif, l'admettent à la forme négative, lutter contre la peur ou l'inquiétude étant une forme d'action susceptible d'un contrôle volontaire. Admettons donc que les passifs de la structure (7), (12b) et (13) contiennent dans leur structure argumentale un agent. Cela permet-il de rendre compte de la différence de sens entre les deux structures 'incapacitatives'?

\subsubsection{Des sens différents dans les deux constructions d'« incapacité »}

On a pu avancer la notion de handicap interne (internally determined capability: Pandharipande 1979), comme spécifique de la construction passive par rapport aux auxiliaires "pouvoir", compatibles eux, aussi bien avec un malaise physique ou mental qu'avec un empêchement extérieur comme avoir un train à prendre ou un rendez-vous urgent. Mais cette notion d'«inner inability " est aussi applicable aux intransitifs en contexte négatif :

(14) dabal calâte mujhse banegâ nahîn, 


\begin{tabular}{|l|l|}
\hline & double conduisant je-instr être-fait-fut neg \\
\hline & hai, harniyâa sabkuch hai \\
\hline & je gros suis diabète est hernie tout est \\
\hline & $\begin{array}{l}\text { je ne peux pas conduire à deux (prendre quelqu'un sur mon vélo) je suis gros, j'ai du } \\
\text { diabète, une hernie, et tout }\end{array}$ \\
\hline
\end{tabular}

La spécificité du passif dans ce contexte apparaît dans les paraphrases de (12b) « ne pas pouvoir se résoudre ", "ne pas se faire à l'idée », alors que celle des intransitifs (12a) marquent la simple inaptitude : «ne pas être fichu de, ne pas être en mesure de, ne pas avoir les moyens de ». L'incapacité interne propre aux passifs, intuitivement perçue comme un bloquage plus fort, relève d'une réticence mentale (c'est plus fort que moi), parfois mise en évidence par des contextes qui métaphorisent cette répulsion terrorisée :

\begin{tabular}{|l|lllll|}
\hline (15) & lekin mujhse jâyânahîn gayâ : pair mâno jamgaehon \\
\hline & mais je-instr aller neg Pass-aor jambes comme se-bloquer allersubj-pft \\
\hline & mais je ne pus faire un pas :j'avais les jambes comme paralysées \\
\hline
\end{tabular}

Tel n'est jamais le cas des intransitifs, dont l'argument instrumental correspond à un rôle assez différent : dans (12a) l'homme qui s'escrime sur la porte n'est pas assez habile ou pas assez fort, alors que dans (12b) au passif il ne peut se décider à ouvrir parce que l'horreur du spectacle qui l'attend derrière la porte le paralyse, ou parce qu'il n'est pas chez lui, ou parce que c'est son premier casse, etc. Le contexte de (12a), où le locuteur demande à quelqu'un de l'aider, explicitant ainsi le trait inaptitude/ inefficacité, interdit la structure instrumentale au passif (12c) :

\begin{tabular}{|l|lll|l|}
\hline$(12) c$ & *bâharse darvâzâ khînclo, sânkal mujhse nahîn kholî jâtî \\
\hline \hline & dehorspar porte tirertiens, chaîne je-instr neg ouvrir Pass-pres \\
\hline & tire la porte de l'extérieur, je ne peux pas ouvrir la chaîne \\
\hline
\end{tabular}

L'intransitif suppose simplement l'inaptitude, conjoncturelle ou constitutive, du participant. Il ne peut pas, comme le passif, enchaîner sur un énoncé contrefactuel du type (16) où le sujet parvient à dominer son aversion intime, exemple un peu tiré par les cheveux, que j'emprunte à Davison (1980) :

\begin{tabular}{|l|lllllll|}
\hline (16) & usse calâ nahîn jâtâthâ, lekin phirbhî vah calâ & \\
\hline \hline & il-instr marcher neg Passimpft mais pourtant il marcha \\
\hline
\end{tabular}


il ne pouvait se résoudre à avancer, mais pourtant il avança

24 «travail faire »), suggérant que la tâche proposée fait horreur, que l'énonciateur ne peut l'envisager sans en frémir (tueur à gages par exemple), et intransitif (de kâm honâ, « être »), pour simplement dire qu'on ne se sent pas à la hauteur ou n'a pas le temps :

\begin{tabular}{|l|l|l|l|l|l|l|}
\hline$(17) a$ & mujhse & yah & kâm & nahîn & kiyâa & jâegâa \\
\hline & je-instr & ce & travail & neg & faire & Pass-fut \\
\hline & je ne pourrai pas le faire (jamais faire ça) (tu me prends pour qui ?) \\
\hline$(17) b$ & mujhse & yah & kâm & nahîn & hogâa \\
\hline \hline & je-instr & ce & travail & neg & sera & \\
\hline & je ne pourrai pas m'en charger &
\end{tabular}

Dans un contexte narratif de mauvaise cohabitation dans un train, pendant une période troublée ou tout le monde se méfie de tout le monde, on trouve dans la même séquence deux emplois dans la structure incapacitative de la même racine verbale uThnâ "se lever ", l'un sur la base intransitive (uThnâ), l'autre sur le passif de la basse transitive uThânâ (lever, soulever). Les deux énoncés peuvent être traduits par «ne pas pouvoir prendre» (il s'agit des bagages à «lever» avant de quitter sa place dans le compartiment) :

\begin{tabular}{|l|l|l|}
\hline (18)a & bibi teri gaThrî main uThâ, lûngâ isgûnge se nahîn uThegî \\
\hline & femme ton baluchon je lever prendrai, cemuet instrneg se-levera \\
\hline & Femme, ton baluchon, je vais le prendre, ce muet est en bien incapable \\
\hline (18)b & mujhse apnâa Trank nahîn uThâyâ jâegâ, na hî ghîkâ Tîn \\
\hline & je-instr refl valise neg soulever Pass-fut neg juste beurre deboîte \\
\hline \hline & je ne pourrai me résoudre à prendre ma valise, pas même le bidon de beurre clarifié \\
\hline
\end{tabular}

(18a) suggère que l'agent (le muet) n'est pas capable de soulever le bagage (trop faible, trop peu serviable) et qu'on ne peut pas compter sur lui. (18b) suggère une forte réticence chez l'agent, terrorisé devant l'air menaçant de son voisin de train: il envisage de changer de place mais se voit dans l'incapacité psychique de prendre sa valise, et même son bidon (qui pèse moins d'un kilo). 


\subsubsection{Sémantique, structure argumentale et morphologie verbale} lexique et de raisonner sur des classes de verbes passivables en fonction des entrées lexicales. Ce qui détermine la possibilité de passivation est la présence d'un argument ayant un rôle d'agent. C'est le cas des verbes transitifs mais aussi de certains intransitifs, où plus justement des contextes syntaxiques où les prédicats se réalisent dans cette structure argumentale.

\section{Autre valeur modale des intransitifs : l'inadvertance}

\subsection{Les faits}

Quand un humain se trouve dans la position de cause inanimée (à l'instrumental) d'un énoncé intransitif affirmatif, celui-ci s'oppose à l'énoncé transitif correspondant comme un processus involontaire à un processus volontaire. On peut ainsi contraster (19) affirmatif à (12)a négatif : 


\begin{tabular}{|l|l|l|l|l|}
\hline$(19)$ & mujhse & gilâs & girâ & TûTâ \\
\hline & je-instr & verre & tomba & se cassa \\
\hline & j'ai fait tomber /cassé un verre par mégarde \\
\hline
\end{tabular}

31 L' 'agent' instrumental, comme la cause animée dénuée par nature d'agentivité consciente et délibérée, s'oppose à l'agent ergatif, doué de ces traits. C'est pourquoi la construction est particulièrement adaptée à la justification des actes répréhensibles (20), le « coupable » se présentant comme irresponsable (21) parce qu'il n'avait pas conscience de ce qu'il a fait, a agi par mégarde, toujours sans le vouloir et souvent sans le savoir, que le prédicat soit un verbe simple ou une locution verbale :

\begin{tabular}{|c|c|}
\hline$(20)$ & yah daftar kâkaTahalhai; mujhse galtî hû̂ \\
\hline & ceci bureau dejacquierest; je-inst faute fut \\
\hline & daftar keahâtemen lagâhuâthâ, mujhse TûTgayâ \\
\hline & Bureau decour dans êtreplantéppft, je-instr secasseralla \\
\hline & -kaise TûTgayâ? - peR par caRhâthâ. \\
\hline & -comment secasseralla? - arbre sur grimperppft \\
\hline & $\begin{array}{l}\text { c'est un jacquier du bureau ; j'ai fait une erreur. Il était planté dans la cour du bureau, je } \\
\text { l'ai cueilli sans faire attention - Comment il s'est détaché /comment tu as fait ton compte } \\
\text { ? }\end{array}$ \\
\hline & - J'étais grimpé à l'arbre \\
\hline$(21)$ & A tumhîn ne uskâ khûn kiyâhai. \\
\hline & - toi-emph erg son sang asfait \\
\hline & B sâhab, mainne khûnnahîn kiyâ, vahto mujhse Hogayâ \\
\hline & - Monsieur je-erg sangneg fis celamais je-instr êtrealla \\
\hline & Khûn mainne jân-bûjhkar kiyâthâ? Vahto mujhse hogayâ \\
\hline & sang je-erg consciemment faireppft? celamais je-instr êtrealla \\
\hline & $\begin{array}{l}\text { C'est toi qui l'as tué. - Monsieur, je n'ai pas tué, ça c'est tué par moi. Aurais-je tué } \\
\text { consciemment? Mais non, c'est advenu par moi (je n'y suis pour rien) }\end{array}$ \\
\hline
\end{tabular}

32 (21) est particulièrement intéressant car A (l'accusation) emploie bien entendu la tournure ergative, que l'accusée nie, n'assumant pas la responsabilité de l'acte mais 
admettant en être la cause, qu'elle exprime à l'instrumental: le bras qui n'est pas un véritable agent, intentionnel et conscient, ne devrait pas être puni.

La construction des intransitifs de sens médio-passif avec un "agent » instrumental introduit donc un argument supplémentaire à la structure argumentale élémentaire du prédicat. Comme il ne peut être un agent, sinon le prédicat serait le transitif corrélé, et que par ailleurs le trait humain le distingue de la cause inanimée ou de l'instrument intermédiaire, il prend les traits sémantiques seuls accessibles dans la construction (volonté -contrôle conscient), qui, ajoutés au trait humain, donnent la non intentionnalité. C'est donc la simple combinaison des traits sémantiques de l'entité nominale et des traits attachés au rôle instrumental dans la structure argumentale du prédicat intransitif médio-passif qui produit ce nouveau type d'argument: l'acteur inadvertant (pour ne pas dire l'agent), dépourvu de contrôle, de volonté et de conscience. De fait, les intransitifs dont l'argument unique n'est pas un patient typique inanimé n'autorisent pas construction ${ }^{13}$, et les transitifs affirmatifs à agent instrumental n'ont pas à la valeur modale d'inadvertance $(1,2)$. La particularité de la construction est donc d'exclure du rôle de source du procès les traits de volonté et de conscience : je l'ai fait sans m'en rendre compte, non seulement sans le faire exprès mais sans en avoir conscience.

\subsection{Instrumental, ergatif, passif et degré d'assomption consciente}

34 C'est en quoi l'instrumental s'oppose clairement à l'ergatif, qui suppose toujours un sujet de conscience même en l'absence des traits de volonté et de contrôle. L'ergatif, comme le passif, correspond bien à un rôle d'agent, mais si on redéfinit l'agent autant par le trait d'assomption consciente que par celui de contrôle volontaire, comme je l'ai montré en le contrastant au datif de l'expérient (Montaut 1991, 1999). Le trait de choix conscient et délibéré invoqué par Butt 1993, et Mohanan (1994: 72-5) est une heureuse manière d'en finir avec la réduction du rôle agent (et du cas ergatif) aux traits du causateur volontaire. Mais il ne suffit pas à rendre compte de sa présence avec pânâ "trouver", mânnâa " considérer ", jânnâ « connaître ", mahasûs karnâa " éprouver ", etc ${ }^{14}$.

L'expression casuelle de l'agent en hindi (ergatif ou nominatif de l'argument principal des transitifs) n'est pas seulement réservée à l'agent canonique pourvu des traits de volonté, contrôle, capacité à déclencher un procès dynamique. Est traité de la même façon sur le plan morpho-syntaxique le sujet de réflexion consciente, ce qui autorise à l'intégrer dans le même super rôle ('Agent') au même titre que les agents canoniques. C'est pourquoi les structures à l'instrumental de l'acteur inadvertant ne se trouvent pas avec des passifs : un verbe passivable comportant nécessairement un des traits de l' 'Agent' au sens large que le hindi associe à ce rôle (fût-il réduit au trait d'assomption consciente), sa structure argumentale ne laisse pas de place à l'acteur inadvertant.

On peut donc pour conclure, souligner l'importance du rôle agent comme condition de la passivation (et de l'ergativité, qui en hindi est déterminée essentiellement par la transitivité), à condition d'intégrer à ce rôle le trait 'sujet de conscience réflexive'. Si les transitifs se passivent c'est non du fait du patient mais du fait de la présence d'un 'Agent'. Les intransitifs qui ne se passivent pas sont ceux qui n'ont pas d'agent à occulter. Quant aux différentes valeurs modales assumées par les passifs et par les intransitifs (inhibition, inaptitude, inadvertance) elles découlent directement de la structure argumentale des prédicats, elle-même liée à leur structure morphologique. 


\section{BIBLIOGRAPHIE}

BRAHIM Ahmed (1996) L'Occultif, hypothèse pour un traitement translinguistique du 'passif' et des structures apparentées, Tunis, Etudes Linguistiques 2.

BUTT Miriam (1993) « Conscious Choice and some light verbs in Urdu », in Complexe Predicates in South Asian Languages, M.K. Verma (ed.), Delhi, Manohar : 31-46.

DAVISON Alice (1980) « Peculiar Passives », Language 56-1 : 42-66.

GAEFFKE Peter (1967) Untersuchungen zur Syntax des Hindi, La Haye, Mouton.

HASPELMATH Martin (1993) « More on the Typology of inchoative/causative verb alternations » in Causative and Transitivity, B.Comrie et M. Polinsky (eds.), Amsterdam, John Benjamins.

MOHANAN Tara (1994) Argument Structure in Hindi, Stanford, Center for the Study of Language and Information.

MONTAUT Annie (1990) « La Construction passive en hindi moderne », BSLP LXXXV : 91-136.

MONTAUT Annie (1991) "Constructions subjectives et constructions objectives », in La Transitivité dans les langues naturelles, A. Montaut (ed.), Linx 24

MONTAUT Annie (1999) « Le datif en hindi », Cahiers de Linguistique de l'INALCO, 2 : 217-24.

PANDHARIPANDE Rajeshwari (1979) «Passive as an optional rule in Hindi, Marathi and Nepali », South Asian Languages Analysis, 1 : 89-106.

SHIBATANI M., (1985), « Passive and related constructions », Language 61-4 : 821-48.

Abréviations : m,f, s,p pour les genres et nombres, nom, acc, dat, erg, instr, gen (nominatif, accusatif, datif, ergatif, instrumental, génitif), pres, aor, pft, ppft, (présent, aoriste, parfait, plusque-parfait), subj (subjonctif), Pass (passif)

\section{NOTES}

1. Type assez rare selon Comrie qui distingue plusieurs types (intransitif dérivé de l'intransitif, comme le se-moyen français, equipollence de verbes non orientés, comme break en anglais, système recourant à deux paradigmes d'affixes sur une même base (communication au colloque Réfléchi et moyen : approches typologiques, Tunis-Manouba, 15-16 mars 2001, org. A.Brahim, avec la collaboration de l'ALT). L'idée d'établir un profil de transitivité des langues par les diverses relations, dérivationnelles ou non, entre intransitifs et causatifs, est d'Haspelmath 1993.

2. Je résume dans cette première section les conclusions de Montaut 1990.

3. Les locutions verbo-nominales intransitives, comme savâr honâ, en-voyage être, «s'embarquer » entrent dans les mêmes constructions : phir savâr huâ gayâ [puis en-voyage être pass-aor-ms] «puis on embarqua »

4. Sur les nombreux problèmes posés par la notion de sujet en hindi/ourdou, voir Montaut 2001. Le fait que le réfléchi soit parfois ambigu, sans jamais exclure l'agent de la coréférence (Mohanan 1994: 123) prouve simplement que l'agent du passif n'est pas un aussi bon candidat à l'antécédence qu'un sujet nominatif ou un actant ergatif et qu'il a moins de propriétés subjectales 
5. Corrélation morphonologique (khulna s'ouvrir, kholna ouvrir, marnâ mourir, mârnâ tuer, pisnâ être écrasé, pissnâ écraser) faisant intervenir un phénomène d'apophonie, ou morphologique ( paknâ être cuit/se cuire, pakâna (faire) cuire, uThnâ se lever, uThânâ lever/soulever, paRhnâ étudier, paRhânâ enseigner, calnâ marcher, calânâ conduire), faisant intervenir un morphème transitivant ou causativant -â suffixé au radical intransitif ou transitif non causatif.

6. A laquelle on peut éventuellement remonter pour ainsi dire à contre courant, en amont (« upstream » dit Langacker). Inversement, le scénario conceptuel dans les procès transitifs (10d) suppose un profilage complet de toutes les phases de la séquence causale (d'amont en aval).

7. Voir l'opposition impératif (transitif) et intransitif : gâkî apne âp nahîn rukegî, ise roko [voiture soi même neg s'arrêtera, elle-acc arrête] 'La voiture ne s'arrêtera pas toute seule, arrête-la'.

8. Mohanan (1994) considère la construction comme «pseudo-passive ». Cependant la morphologie verbale est commune (la catégorie de voix étant fondamentalement morphologique). Certes, l'auxiliaire jânâ «aller » a d'autres emplois que de former les passifs en hindi moderne, mais en sanscrit, le morphème spécifique -ya- qui sert à passiver les transitifs sert aussi à passiver les intransitifs, et en hindi ancien (dit « médiéval ») cette passivation, encore synthétique ou périphrastique, dans le même environnement (négatif, instrumental), produit la même valeur modale (Gaeffke $1967: 39-58$ )

9. Exemple en contexte virtuel : mujhse jo kuch banâ, kiyâ ; jahân tak mujhse banâ, kiyâ je-instr rel indef être-fait-aor, faire-aor-ms ; jusqu'à ce que je-instr être-fait-aor, faire-aor «tout ce que je pouvais faire, je l'ai fait; je suis allé jusqu'au bout de ce que je pouvais faire » 10. La construction modale traite toujours l'instrumental comme seul antécédent du réfléchi (Mohanan 1994 : 161), alors que la construction non modale peut tolérer l'ambiguïté (voir note 4). bahut prayâs karke bhî usse apnâ pâTh paRhâ nahîn jâtâ beaucoup effort faisant même il-instr refl leçon étudier neg pass-prest-ms «en dépit de tous ses efforts il n'arrive pas à apprendre »

11. Un examen exhaustif serait à faire et on constaterait certainement des « exceptions » à cette généralité : on peut avoir ruko (impératif se ruknâ « s'arrêter ») et non son passif. De même âo! «viens! » est parfait mais son passif hautement improbable.

12. darvazâ havâ ke jhonke se khulâ porte vent de coup .instr. s'ouvrit : 'un coup de vent ouvrit la porte'

13. * mujhse cillâyâ gayâ je instr crier passif-aor (au sens 'un cri m’a échappé').

14. maine sîtâ ko akele rote hue pâyâ

je-erg Sita acc seule pleurant étant trouver-aor

'j'ai trouvé Sita en train de pleurer toute seule'Sitâ ko akele rote hue pâyâ gayâ

Sita acc seule pleurant trouver Pass-aor

'Sita a été trouvé pleurant seule', 'on a trouvé Sita en train de pleurer toute seule'

\section{AUTEUR}

ANNIE MONTAUT 